\title{
Making Associativity Operational
}

\author{
Amir H. Asghari ${ }^{1,2}$ • Leyla G. Khosroshahi ${ }^{2}$
}

Received: 22 February 2016 / Accepted: 18 July 2016 /Published online: 4 August 2016

(C) The Author(s) 2016. This article is published with open access at Springerlink.com

\begin{abstract}
The purpose of this paper is to propose an operational idea for developing algebraic thinking in the absence of alphanumeric symbols. The paper reports on a design experiment encouraging preschool children to use the associative property algebraically. We describe the theoretical basis of the design, the tasks used, and examples of algebraic thinking in 5-6-year-old children. Theoretically, the paper makes a critical distinction between operational and structural meanings of the notion of equality. We argue that mathematical thinking involving equality among young learners can comprise both an operational and a structural conception and that the operational conception has a side that is productively linked to the structural conception. Using carefully designed hands-on tasks, the crux of the paper is the realization of algebraic thinking (in verbal mathematics) as operationally experienced in the ability to transform one number structure, with a quantity that is subject to change, into another through equality-preserving transformations.
\end{abstract}

Keywords Associative property Early algebra Operational algebra Preschool . Structural algebra

There is a growing consensus that "elementary students can learn to think about arithmetic in ways that both enhance their early learning of arithmetic and provide a foundation for learning algebra" (Carpenter, Levi \& Farnsworth, 2000, p. 1). This study extends the existing research by investigating the possibility of developing algebraic thinking in the absence of alphanumeric symbols at the preschool level. In particular, we focus on the associative property that is one of the main components of mental and

Amir H. Asghari

Asghari.amir@gmail.com

Leyla G. Khosroshahi

1_khosroshahi@yahoo.com

1 School of Education, Liverpool John Moores University, Liverpool L17 6BD, UK

2 Mathematics Department, Shahid Beheshti University, Daneshju Blvd, Tehran 19839 63113, Iran 
written methods of calculations (McCallum, Zimba \& Daro, 2011). However, in the absence of writing symbols (namely, alphanumeric, symbols for addition and subtraction, equality symbol, and parentheses), careful attention should be paid to the meaning and the means of bringing about such an understanding. This paper does so while reporting a design experiment, its theoretical background, and learning activities. Moreover, we show how some preschool children (aged 5-6) experienced the algebraic nature of the associative law within the situation designed.

\section{The Algebraic Nature of Arithmetic Facts}

An important part of so-called early algebra has been to investigate the extent of children's awareness of a certain general fact demonstrated in a specific arithmetic problem. For example, if students explain that the equality $78-49+49=78$ is true "because you took the 49 away, and it's just like getting it back," it might be inferred that

Although the students used a specific example to justify the generalization, the way they explained the example showed that they understood that they could do the same thing with any numbers. (Carpenter et al., 2000, p. 3)

To bring about such an understanding, Fujii and Stephens (2001) propose the use of quasi-variables, by which they mean numbers that vary within "a number sentence or group of number sentences that indicate an underlying mathematical relationship which remain true whatever the numbers used are" (p. 259). For this approach to work, one needs to be or to become a "proceptual thinker" (Gray \& Tall, 1994) who can resist immediate direct calculations and an operational conception of the equal sign (Kieran, 1981) and concurrently realize which particular features should be stressed and ignored. Only then would it be possible to see a specific equality like $78-49+49=78$ as a generic equality and "the carrier of the general" (Mason \& Pimm, 1984) in which both 49 and 78 can be understood as a quasi-variable indicating that the sentence belongs to a type of number sentence. Put simply, one needs a structural conception of the equal sign. In this paper, we propose a different approach that does not rely on such a conception.

\section{Operational and Structural Sides of Equality}

The equal sign is a symbol to indicate the notion of equality, and equality tells us whether two mathematical objects, possibly presented to us in wildly different ways, are to be considered equal (Mazur, 2008). Strictly speaking, the equal sign represents equivalence. The number expression $8+2+4$ is equivalent to (or "is the same as") $(8+2)+4$ or $8+(2+4)$. Students can use some structured materials, say Cuisenaire rods, to model the number expressions and see either way they would end up with the same number of blocks, that is, $(8+2)+4=8+(2+4)$. We call this focus on the equality as equivalence, the structural conception in which we see the both sides of the equal sign 
intact (Carpenter, Levi, Franke \& Zeringue, 2005; Stephens, 2006; Molina \& Ambrose, 2008; Stephens, Knuth, Blanton, Isler, Gardiner \& Marum, 2013).

However, when students transform $8+6$ into $10+4($ i.e. $8+(2+4)$ into $(8+2)+4)$ and then to 14 , the focus is on the transformations that have taken place while intermediary structures might "have only transient existence" (Caspi \& Sfard, 2012, p. 50) in the process of calculations. We call this focus on the equality as transformation, the operational conception in which we replace one number expression with another through "equality-preserving transformations" (Kieran, 2014) without necessarily paying attention to the expressions for their own sake. Both conceptions of equality can play a productive role in "thinking relationally about equality" that is one of the main themes of "algebraic thinking and the routes by which its growth might be encouraged” (Kieran, 2014, p. 1) in arithmetic. However, it is not an easy route.

\section{With Letters It Is Hard, with Numbers It Is Even Harder}

Based on historical and empirical evidence, Caspi and Sfard (2012) argue that the first level in the development of algebra is processual, where the focus is on numerical calculations in the order of their execution on constant (or as in Sfard \& Linchevski (1994), fixed) values, either known or unknown. With letters, it would be challenging to break away this default linear structure and with numbers even more challenging. Arithmetic is where the so-called left-to-right approach commonly used in early years of school (Booth, 1989; Kieran, 1989) goes hand in hand with the students' "tendency to think about expressions involving binary operations in terms of a sequential procedure" (Larsen, 2010, p. 42). Accordingly, both expressions $8+2+4$ and $7+$ $9+1$ have the same chance of being treated from left to right, as $(8+2)+4$ and $(7+$ $9)+1$ regardless of the addends involved. Arithmetic is where the operational conception of the equal sign is most frequently realized as instructions to do something and as an obstacle for the structural conception, that is, equality as equivalence (Kieran, 1981). Accordingly, the response to the open number sentence $8+6=\square+4$ would be 14 (Stephens et al., 2013).

Yet, arithmetic is where we wish to cultivate students' relational thinking about equality. One way to do so is to bring out the relational meaning of the equal sign whenever it is used. However, it is doubtful that simply telling students what the equal sign means effectively develops understanding (Carpenter, Franke \& Levi, 2003). Instead, we might use a structural program in which students see structural expressions and equations in their entirety. For example, we might develop a set of true/false (e.g. $(4+7)+9=4+(7+9)$ ) or open number sentences (e.g. $56+75+25=\square)$ that might encourage our students to experience and to make explicit the associative property (Carpenter et al., 2003; Carpenter et al., 2005). In this way, students may find a correct operational sense of when and how it is appropriate to use the associative property to transform one mathematical expression into the other. However, when working with prealphanumeric students and in the absence of writing symbols to record number sentences, a structural program would be hardly accessible. Yet, we might encourage an operational conception of equality. This paper proposes an approach to do so, using the associative property as an example. 


\section{The Associative Property}

It is common to think of $(a+b)+c=a+(b+c)$, but not $(a+b)-c=a+(b-c)$, as the associative property. However, using the concept of additive inverse, we can see $(a+b)-$ $c$ as $(a+b)+(-c)$, and then as $a+(b+(-c))$, which is the same as $a+(b-c)$. The same argument applies to $(a-b)+c=a+(c-b)$ and $(a-b)-c=a-(b+c)$. Of course, this way of looking at associativity is too advanced for a preschooler or a primary schooler. However, children can use these equalities when operating with natural numbers and without thinking of the concept of additive inverse. For example, while transforming $13-$ 5 to $13-3-2$ by decomposing $5=3+2$ (with respect to 10 ), one implicitly replaces 13 $-(3+2)$ with $(13-3)-2$. The equality $13-(3+2)=(13-3)-2$ provides two different ways of structuring and restructuring $13-3-2$. In general, the same thing can be said about $(a+b)+c=a+(b+c),(a+b)-c=a+(b-c),(a-b)+c=a+(c-b)$, and $(a-b)$ $-c=a-(b+c)$ in relation to $a+b+c, a+b-c, a-b+c$, and $a-b-c$, respectively.

Moreover, the associative property can also be translated in the language of transformations. Consider that $\pm b \pm c$ "can be perceived as a composition of two transformations that yield a third transformation" (Peled \& Carraher, 2008, p. 320). Thus, one might move from $4-2+1$ to $4+(-2+1)$ (where " -2 " is thought of as a change), and then, to $4-1$ as an attempt to figure out the total change that has been applied to the stating number (here, 4). In this paper, the focus is on $a+b-c$ and $a-b+c$, interpreted as adding-removing and removing-adding, respectively.

\section{The Purpose of Study Revisited}

The purpose of this study was to investigate the possibility of developing an algebraic understanding of the associative property in the absence of alphanumeric symbols. Our first step was to interpret associativity operationally, in terms of transformations. In this respect, the aim was to help children to avoid the temptation of calculating from left to right and instead encourage them to restructure the given expression. For example, we intended that children be able to interpret $3+4-2$ as $3+(4-2)$, rather than automatically as $(3+4)-2$. Learning to restructure an arithmetic expression is an important step towards relational understanding of equality in algebra. However, such restructuring only involves known specific numbers in a resultoriented calculation. In contrast, one essential component of algebraic thinking is the ability of dealing with the indeterminate quantities that are unknowns or variables (Radford, 2014).

Hence, we had to address two problems. To address the first problem (i.e. total change), we adopted the didactical strategy of dissuading the learner from touching (i.e. calculating with) the starting number (Asghari, 2012), hoping that it also addresses the second problem (i.e. indeterminacy). For example, working with the expression $3+4-2$. If we prevent the actualization of the initial and subsequent operations ( $3+4$ and $7-2$ respectively) and instead invite children to operate on the starting number (here, 3 ) while leaving it intact, then, they might transform 3 $+4-2$ to $3+(4-2)$, and then, to $3+2$. In doing so, the specific starting number (here, 3 ) might turn into what is called a specular number that is specific for the learner, but it is treated as a non-specific number (Asghari, 2012). As such, one of 
the operands (here, 3) is treated as an indeterminate value, while the problem is restructured into a new one through equality-preserving transformations.

The idea of specularity seemed promising since its focus is on "the process acted on the object" (Asghari, 2012, p. 37) rather than the structural features of the problem. Whether it works in the absence of writing symbols was the subject of a one-on-one design experiment (in the sense of Cobb, Confrey, diSessa, Lehrer \& Schauble, 2003) of which the research question was as follows.

In preschool children ages 5-6 years and in the absence of alphanumeric symbols, can the idea of specularity promote an operational algebraic understanding of associativity?

\section{Methodology}

We conducted a series of sessions with a small number of preschoolers. The sessions were test-beds for practical variations of the idea of specularity as a means to promote operational algebraic understanding (of associativity). Preschool children who participated in the study were from the same preschool in the north of Tehran. In Iran, educational programs at the preschool level vary from one preschool to another. In this particular preschool, the focus of their program was mostly on hands-on activities. As far as mathematics is concerned, their focus was on counting activities and the wellknown activities of trying to find a one-to-one correspondence between sets and hence to show whether the sets have the same cardinality or not. Since numeral recognition and numeral writing is part of the centered national mathematics program for the first year of school, the preschool program was not obliged to reach any particular target in that direction; at the best, their point was to create a sense of familiarity with numerals. We also did not use numerals in the study.

The study had two main cycles in which two different groups of students participated. The first group consisted of nine children. The first cycle was the developmental stage of the study in which different tasks were designed and tried out. A year later, we worked with a different group consisted of five children. Now, the study was in a more advanced stage and only a carefully chosen number of tasks were used to get the desired outcome. More importantly, as a result of our growing understanding of the situation, a fundamentally new task was added to the previous tasks.

A number of individual task-based interviews were used in both cycles. Each interview that lasted between 5 and 15 min (once a week) was videotaped and subsequently transcribed for further analysis. The maximum number of interviews with each child (done by Leyla, known to children as auntie Leyla and one of the authors of the present article) was 20 in the first cycle and 7 in the second cycle. Generally, in both cycles, the first few tasks were numeric. The tasks (not reported here because of space limitations) included counting a number of objects (toys, counters, etc.) and one-step addition and subtraction problems with or without hands-on material. The aim of the numeric tasks was to decide the range of numbers that would be meaningful to each child. In the main stage of the study, we used five types of tasks, all being variations of the same theme: structuring/restructuring a to-be-specular number sentence with two consecutive operations on a number intended as a specular number (specific, but untouchable). In task 1 (hereafter, "task $x$ " is representative of its type), the starting 
number was known and "small", in task 2, known and "big", in task 3, unknown and specific, and in task 4, known and small again; finally, in task 5 (added to the study in the second cycle), we used two tasks simultaneously with two unknown starting numbers. The details and reasons behind all these choices will be explained below. The following data come from both cycles of the study.

\section{The Study}

\section{The First Cycle}

Structure of Tasks, Illustrated on Task 1. There are two small toy animals, a frog and a ladybird, and two empty boxes (Fig. 1). Each toy is attached to one of the boxes. Leyla counts three candies and places them in the frog's box. She does the same for the ladybird's box. Then, Leyla counts four new candies and adds them to the frog's box, and finally, she visibly removes two candies from the same box. Although the child is allowed to see the whole process and to know the beginning number of candies in each box and the number of candies added and removed, it is not allowed to see or touch (say, for counting) the content of the two boxes, unless it is asked for by the child. The task for the child is to determine which toy has more candies.Various variations of the task above were designed and used with different background stories, hands-on materials, and numbers and yet similar underlying idea. Generally, each task started with two "number holders" initially holding the same number of objects. The starting number could be known and "small" (task 1 and task 4), known and "big" (task 2), or unknown (task 3 and task 5). The starting number was intended to be a specular number, simply by hiding the (number of) objects, say, inside a box. Hereafter, square brackets around the starting number indicate its intended role (e.g. [3] in task 1). Then two operations in which what being added or subtracted was in the range of 1 to 10 , one after the other, were applied on one of the holders (e.g. the frog's box in task 1). The problem was to compare the number of objects of the holders with each other (e.g. [3] $+4-2$ and [3] in task 1). In this paper, the pairs of operations are adding-removing and removing-adding. In relation to the design of the tasks, there are two points that should be highlighted.

First, in the absence of writing symbols to record the problem, it is not easy to track which number is compared with which number. For example, in the task above, if we

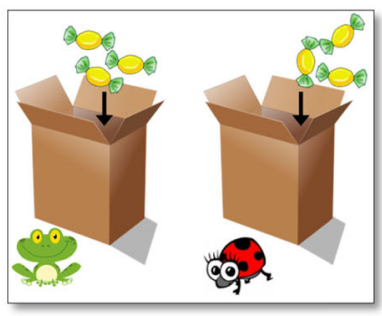

(1)

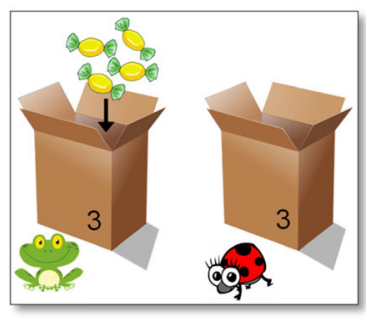

(2)

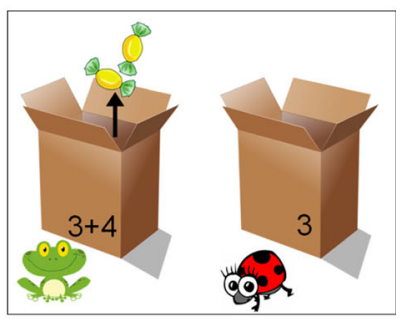

(3)

Fig. 1 Task 1 
had used only one box, it would not have been clear for the child whether the final amount ([3] $+4-2)$ should be compared with the intermediate amount $([3]+4)$ or the initial amount ([3]). The use of two different boxes reduces such ambiguities. Moreover, the presence of two number holders could keep the starting number specific (though, untouchable) during the whole process and also allow the child to check his or her answer (if necessary).

Second, the tasks ask for the comparison of the initial and final states, not for the calculation of the final result. Asking for the final result would be a direct invitation to the left-to-right calculation when starting with a known number (task 1) and meaningless when starting with an unknown number (task 3).

It is now time to see the tasks through children's eyes.

Task 1: the Starting Number Is Known and Small. Task 1 is one of more than hundred tasks with a known and "small" (yet, untouchable) starting number that were used in both cycles of the study. This version of the tasks allowed for various solutions as follows (notice that though the term left-to-right is reminiscent of written calculations, for simplicity, we sometimes use it to refer to the order imposed by the presentation of the tasks).

1. Following the left-to-right order that includes moving from $([3]+4)-2$ to $7-2$ and then to 5 which is subsequently compared with the initial number (3). Here, the focus is on the result of each operation one after the other.

2. Changing the structure of the task from $([3]+4)-2$ to $[3]+(4-2)$ and then following one of these three options: (i) calculating the total change $(4-2=2)$ and then applying it to the initial number $(3+2=5)$, which is subsequently compared with the initial number (3); (ii) calculating the total change $(4-2=2)$ and realizing that it is a "positive" change: [3] +2 is more than [3]; (iii) realizing the direction of change (whether it is "positive" or "negative") without calculating the amount of change: the number added (4) is more than the number removed (2), thus the final is more than the initial. In many cases, children could not explain how they have solved the task at hand. For example, Melina (a 5-year-old girl) said: "this ([2]+2-1) has more; it has three, but that one has two." When Leyla asked: "how do you know?" she answered: "I just know." When the calculations were a bit more cumbersome, there was more chance to observe the strategy used. The following excerpt illustrates how Sarah (a 6-year-old girl) used the left-to-right strategy to solve task 1:

Sarah: May I use my fingers?

Leyla: Yes, of course.

Sarah: Three plus four is seven [she held up three fingers on her left hand and four fingers on her right hand. Then she counted all the fingers held up]. Then you removed two [she closed two fingers out of four held-up fingers on her right hand and counted all the fingers that remained up on both hands]. It's five.

Leyla: So which toy has more candies? 
Sarah: Frog.

There were also cases that the child could explain how the task has been solved. For example, Inez (a 6-year-old girl) used her fingers in a left-to-right calculation to compare $5-2+3(=6)$ and 5 . But, when faced with [6] $+3-2$, she explained her strategy:

Inez: This $([6]+3-2)$ has more; it's seven.

Leyla: How do you know that it's seven?

Inez: Because, you added three, and took away two.

She stresses on number 2 meaning that it is less than the number added. Then, she proceeds by applying the total change $(3-2=1)$ to the initial number $6+1=7$.

Inez's change of strategy from one task to the other was typical of this stage of study in which children worked with known starting numbers. The same child could solve each task by either of the two strategies, whether the task was adding-removing or removing-adding. However, the available evidence from more than a hundred tasks suggested that the size of the numbers influences the child's strategy. For example, Inez could easily find the result of [5] $-2+3$ from left to right by calculating the result of 5 $-2(=3)$ and then the result of $3+3(=6)$. The left-to-right approach to [6] $+3-2$ would lead to $6+3(=9)$ and $9-2(=7)$ which both were beyond the calculating ability she showed earlier when answering the numeric tasks.

It is important to notice that "number size" is a relative concept. Thus, while the magnitude of the left-to-right calculations in [6] $+3-2$ was big enough to lead Inez to restructuring the problem, even something like [4] +6-5 could not hinder Parsa (a 6year-old boy) from using the imposed structure of the task. True, the presentation of the tasks did not allow children to use physical objects to represent and solve the problem, but it did not stop them to model the problem on their fingers or mentally carry the calculations when the numbers involved were "small" enough for them. In general, the more a child was able to operate on the initial number, the more he or she was inclined to apply the imposed structure of the task. Thus, for more able children, the known starting number (though untouchable) was more specific and less specular. For these children (like Parsa), the tasks were just an arithmetic addition and subtraction task. For those (like Inez) who were at times impelled to restructure the problem (say, from $([6]+3)-2$ to $[6]+(3-2))$, restructuring was mainly directed towards finding the final result of the numeric sentence. In a way, the restructuring was attached to the starting number. The aim of the next two tasks was to bring the restructuring to the fore, receding (calculations with) the starting number into the background.

Task 2: the Starting Number Is Known and Big. In task 2, the magnitude of the starting number and the middle and final results is "big" enough to block the imposed order of the calculations and encourage the use of restructuring strategy. Here, the candies of a bag of a hundred candies are emptied into a box. Leyla adds three candies to the box and then removes four candies from the box (Fig. 2). 
Leyla: Are there more or less than hundred candies in the box?

Sarah: Oh, it's very easy. It is less than a hundred.

Leyla: Why?

Sarah: You put three [candies] in place of that four [candies], and so, "hundred" went down one.

Leyla: What should I do if I want it to be a hundred again?

Sarah: You should add one.

In using a big number, Sarah's inability to model the problem on her fingers turned into the ability of perceiving $(100+3)-4$ as $100+(-4+3)$ and then as $100-1$ (here, $-4+3$ represents a composition of two transformations).

A big number would work providing that the child could apprehend it as a specific whole. Thus, while 20 (and for some children, even 10) was big enough to encourage restructuring, a number like 100 could be too big. There were times that children comprehended a "big" number as "too many" (rather than as a fixed whole), where a bit more or less than "too many" was still "too many"! In the following excerpt, Fatima (who was competent with numbers in the range of 1 to 10 ) compares $100+3-2$ with 100 :

Fatima: It's more than hundred.

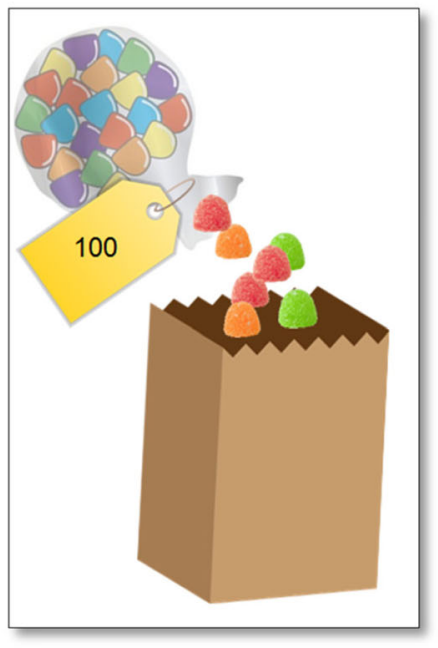

(1)
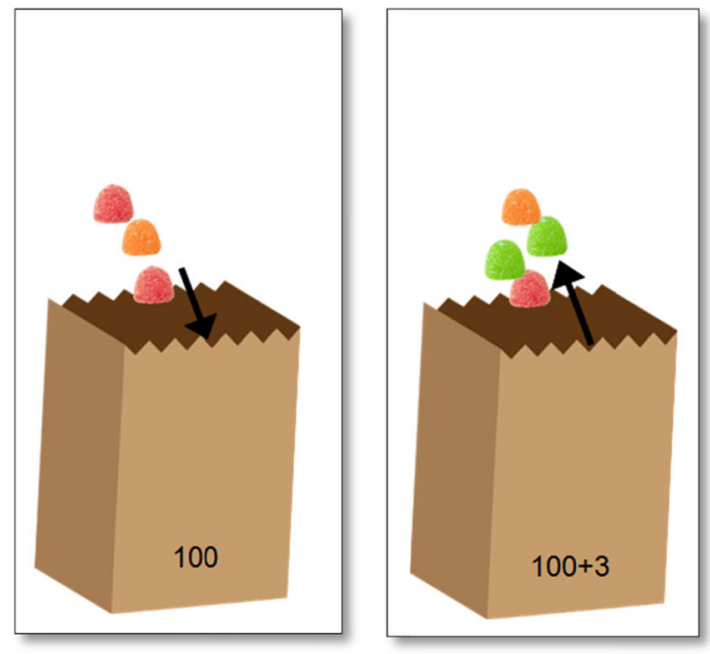

(2)

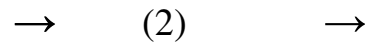

(3)

Fig. 2 Task 2 
Leyla: Why?

Fatima: Aha! It's less than hundred!

Leyla: Do you remember what I did?

Fatima: Yes, you added three [candies], and then, removed two [candies].

Leyla: How many candies were inside the box?

Fatima: Hundred.

Leyla: Now, how many candies are inside the box?

Fatima: Twenty!

A "big" number, if meaningful to the child, could encourage the change of structure while leaving the starting number intact. However, again, the transformations are bound to a specific number (say, 100), at least for us as observers. The next task is to address the indeterminacy of the starting number.

Task 3: the Starting Number Is Unknown and Specific. Here, the starting number is big enough that cannot be subitized and small enough that can be counted and used in calculations (if allowed or wanted). Here, there are two equal height towers (with the same number of cubes), one red and the other blue. Leyla removes one cube from the red tower (puts it on the table) and adds three cubes to the same tower. Sarah does not see the towers, but she sees that one red cube comes out from behind a pad and then three red cubes go behind the pad (Fig. 3).

Leyla: Which tower is taller now?

Sarah: Red has two more.

Leyla: How do you know?

Sarah: Because one of those three [cubes] replaces the one you removed; and the other two [cubes] make the red tower taller than the blue tower.

With an unknown starting number, there is no other way to solve the task but by restructuring it. Sarah restructured the initial arithmetic structure of the task from $([a]-1)+3$ to $([a]-1)+(1+2)$ and then to $[a]+2$. This is decomposing $3=1+2$ with respect to the number taken away and then using the associative law twice: first in restructuring $([a]-1)+(1+2)$ to $(([a]-1)+1)+2$, and second, in transforming $([a]-1)+1$ to $[a]$. The second transformation can be also thought of as making use of "the principle of inversion" which is known to be used by children even younger than Sarah (Geary, 2006). 

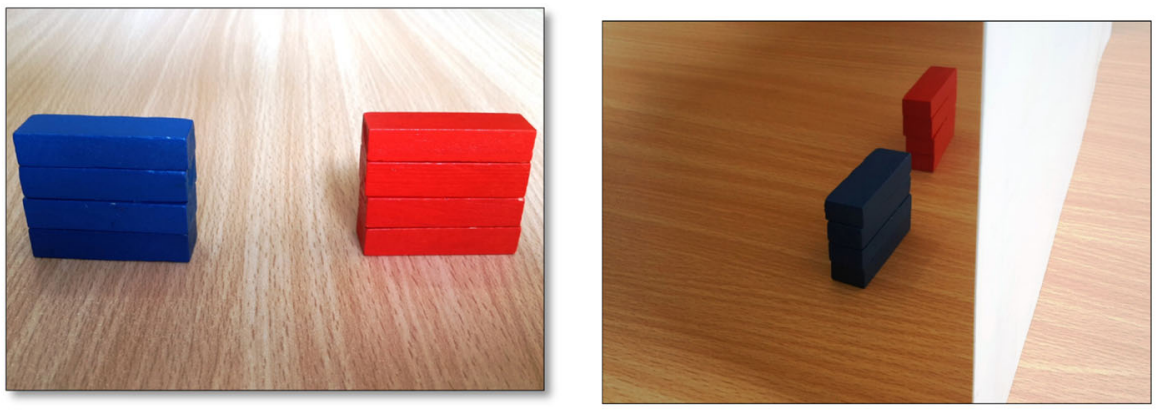

(1)

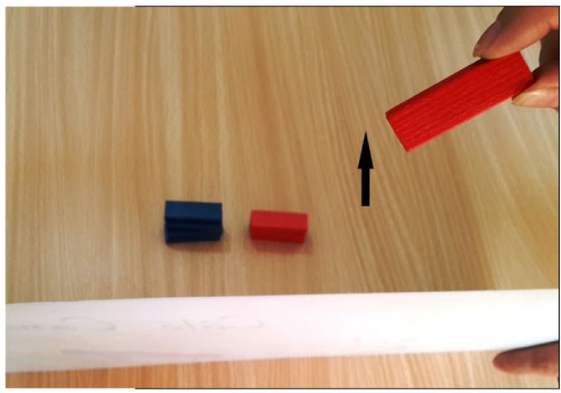

(3) $\rightarrow$

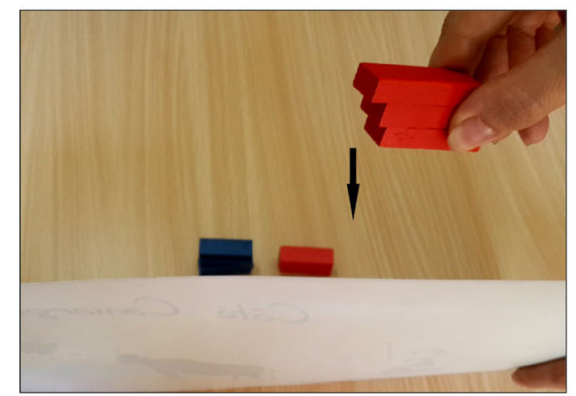

(4)

Fig. 3 Task 3

Sarah spontaneously answered two questions: "which one has more?" and "how many more?" Most children (including Sarah), most of the times, just answered the former question, mentioning the direction of the total change, without calculating the amount of the total change. Thus, a common answer could be something like, "Red is more because you removed one, but added three." In fact, for most children, the difference questions ("how many more?") were hard even for two known numbers, let alone two unknown numbers (say, $[a]$ and $[a]-1+3$ in the task above). For two known numbers, the typical answer was to say the bigger number (see also Hudson, 1983). For two unknown numbers (task 3), if they were asked the question, the typical answer was, "I don't know because we didn't see inside it." However, if the question was, "How many must we add to this box ([a]) so that it will have the same number as this box $([a]-1+3)$ ?" then it was more likely to get a correct answer. Phrasing the difference question in terms of transformations made it like an equalizing problem in arithmetic where "the question is directly about how much to add to one set to make it equal to the other set" (Nunes \& Bryant, 1996, p. 130). Unlike arithmetic problems, the situation here is not start-known and result-known. However, it is not quite startunknown and result-unknown either. The starting number and accordingly the final number, both are intended as specular numbers. They are unknown but could be 
disclosed by the child whenever wanted. As such, children could think of "this number" (the number of objects in the box), though it was unknown (Asghari, 2012; Radford, 2010). More importantly, they could operate on one possible value for the number holders.

Parnian (a 6-year-old girl): [Skillfully modeling 4-3 on his fingers while comparing blue tower $([a])$ and red tower $([a]+4-3)]$ Red is one [cube] taller.

Leyla: Can you tell me how many are the reds?

Parnian: [After pausing a few seconds] No, I can't. I didn't see how you built the towers.

Leyla: Okay. I give you a clue. The number of blues is ten.

Parnian: [Immediately] Eleven.

Even sometimes, they could operate on more than one possible value.

Sarah: If this $([a]-3+2)$ is nine, this $([a])$ is ten.

Leyla: And if this $([a]-3+2)$ was five?

Sarah: Six.

Whether thinking of possible values (one at a time) is an indication of the generality perceived by the child is the subject of the discussion below and the second cycle of the study. For now, it is important to say that task 3 makes the child aware of the possibility of the change of structure by forcing the change. Whether the child applies such restructuring in the absence of such a force is the subject of task 4 .

Task 4: the Starting Number Is Known and Small. The starting number is small and known. The whole expression is within the calculating ability of the child. However, now, unlike task 1 , the child has experienced several restructuring tasks. Here, the task was to compare [4] +4-1 (red cubes) with [4] (blue cubes).

Leyla: Which group has more cubes?

Sarah: The red one. It has three more cubes [than the blue group].

Leyla: How many cubes does it [the red group] have now?

Sara: [while using her fingers to add 3 to 4 ] it has seven.

Without restructuring, the order of approaching the task is to calculate the final answer according to the imposed order of the task and then compare it with the starting number (see Sarah, task 1). With restructuring, the total change can be calculated first, 
and then, the total number (if needed). This is what Sarah did when the initial arithmetic structure of the task was changed from $([4]+4)-1$ to $[4]+(4-1)$ and then to $4+3$. This is also what Parsa did when he calculated [6] $+3-2$ at this stage of his work, while earlier, he had solved all the start-known problems with "small" numbers by direct calculations from left to right (see task 1 above). What they did is an indication of awareness of the possibility of the change of structure. However, it is not an indication of a free choice of strategy. It is more likely that the similarity of the presentation of the task with the previous ones (in particular, the unknown-number version) somehow triggered what they did. We will discuss this point further in the next section.

\section{Discussion}

The didactic strategy used in the current study was making the starting number untouchable during certain known operations. Accepting an untouchable number would not be as easy as it looks in our reports above. In fact, there were many occasions, before and after getting used to the idea, where children felt obliged to make the number touchable to be convinced about the correctness of the answer by counting the objects inside each box. Thus, when the starting number was untouchable but knowable and representable on the fingers (task 1), the child's approach to the task was hardly predictable. Overall, it seems that if applying the imposed order of the problem were feasible, the child would choose the left-to-right approach. Sarah and Parsa successfully solved several of such problems by direct calculations at the start of the interview process. Thus, the change of their strategy (task 4) could be due to their exposure to the previous tasks (say, task 3). However, such tasks could make the child aware of the possibility of the change of structure, but not necessarily of the suitability of such a change for the problem at hand. True, task 3 forces the child to restructure the problem whether it is [6] $+4-1$ or [7] $+4-3$. However, restructuring $6+4-1$ as $6+(4-1)$ does not necessarily make the calculations easier for the child (if it does not make it harder). In arithmetic, the proper use of associativity is a decision that is taken based on each and every three operands and the numeric relations they have together. It is based on such relations that a person decides to restructure one expression but not the other. To provide such awareness, there needs to be a different strategy allowing children to reflect on different ways of structuring the same number expression. This is where a structural approach (e.g. Carpenter et al., 2003) might come handy. In a way, our children learned to restructure without necessarily seeing the structures.

\section{Introduction to the Second Cycle}

Here is the outline and the outcome of the first cycle of the study.

Task 1. [6] $+3-2$ could impel the child to restructure it as [6] $+(3-2)$. However, the starting number was so attached to the problem that something like [4] $+3-2$ could be considered as a new problem, not the same problem as [6] $+3-2$ with a different starting number. 
Task 2. A big starting number could bring the change of structure to the fore. However, the whole structure could also collapse because children could miss the numerosity of the starting number. The starting number was meant to be a specular number, a specific number for the learner that is begging to be treated as a non-specific number of its kind (Asghari, 2012).Of course, it would not play its intended role if it misses its specificity. Task 3 was to resolve this problem.

Task 3. In $[a]+3-2$, the number of the objects in the box is not known to the child. However, he or she could apprehend it as a specific whole and disclose the number whenever wanted. The presentation of the task forces restructuring [a] $+3-2$ to $[a]+(3-2)$ and then to $[a]+1$. As the result of transforming $([a]+3)-2$ to $[a]+1$, part of the structure (here, $+3-2$ ) collapses, but an important part remains unchanged (here, [a]) and the initial structure is operationally transformed into an equal structure. Moreover, transforming $([\mathrm{a}]+3)-2$ to $[\mathrm{a}]+1$ indicates a change of structure in which neither of the structural expressions $([\mathrm{a}]+3-2,([\mathrm{a}]+3)-2$, $[\mathrm{a}]+(+3-2)$, and $[\mathrm{a}]+1)$ necessarily stands alone. In the productive sense of the operational conception, such an equality-preserving restructuring is algebraic, since it is a trigger - and an indication - of a relational conception of equality (which might be applied to an arithmetic problem: task 4). However, the problem that we started with is still present. The question is to what extent the child who has experienced such transformations also experiences [a] as something that represents any number (of objects inside the box). From the "big" starting numbers (task 2), we know that even if the child moves from "one" to "any", from one specific number to a set containing that number, the set is restricted to the numbers whose numerosity can be experienced by the child. From the unknown starting numbers (task 3), we know that children can think of one (or in some cases, more than one) possible value (one at a time) for the starting number, but not whether the child perceives the specific initial number as a single, but indefinite, whole number (Mason \& Pimm, 1984). The problem is how we can discern the extent of the generality perceived by the child "when any instance is of a constant, unvarying nature" (Carraher, Brizuela \& Schliemann, 2000, p. 150) - in short, how we know that the initial specific number has played its role as a specular number; hence, the second cycle of the study.

\section{The Second Cycle}

The first cycle of the present design experiment clarified the notion of operational algebra as equality-preserving restructurings. However, as far as algebra is concerned, what is being done is being done on a quantity that is subject to change; this involves a simultaneous awareness of what is being done and what is being changed. Though the problem of assessing such awareness is notoriously difficult (Carraher et al., 2000; Mason, Stephens \& Watson, 2009), the main distinguishing feature of the second stage of the study is to offer a solution. Task 5 was given after all the first four tasks had been completed.

Task 5: the Starting Number Is Specular. There are two toys; each one has two boxes in two different colors (Fig. 4). The boxes of one of them are blue and red; both 
boxes contain the same unknown number of foods, say [x]. The boxes of the other toy are green and yellow; both boxes contain the same unknown number of foods, say [y]. These two unknown numbers, $[x]$ and $[y]$, are not necessarily equal to each other. Leyla chooses the red box (of the first toy) and the green box (of the second toy), adds four foods to each one of them, and then removes two foods from each one of them. Each toy only has access to its own boxes, and each toy chooses the box with more food. The task is to determine which box each toy chooses:

Helen (a 6-year-old girl): They choose Red and Green.

Leyla: How do you know?

Helen: Because you added four to them and removed two from them.

Using each one of the tasks alone (like task 3), we would have had another indication of the child's ability of relational thinking. Could we interpret this as an indication of the child's awareness that in the transition from $([a]+4)-2$ to $[a]+2$, the starting number can vary? By the definition of specularity, the answer could be "yes." However, our students do not learn because of our definitions! There is a possibility that in every single task, the specular starting number is mentally fixed, hence, at the best, an indication of relational arithmetic. Task 5 was set up to try and rule out this possibility.
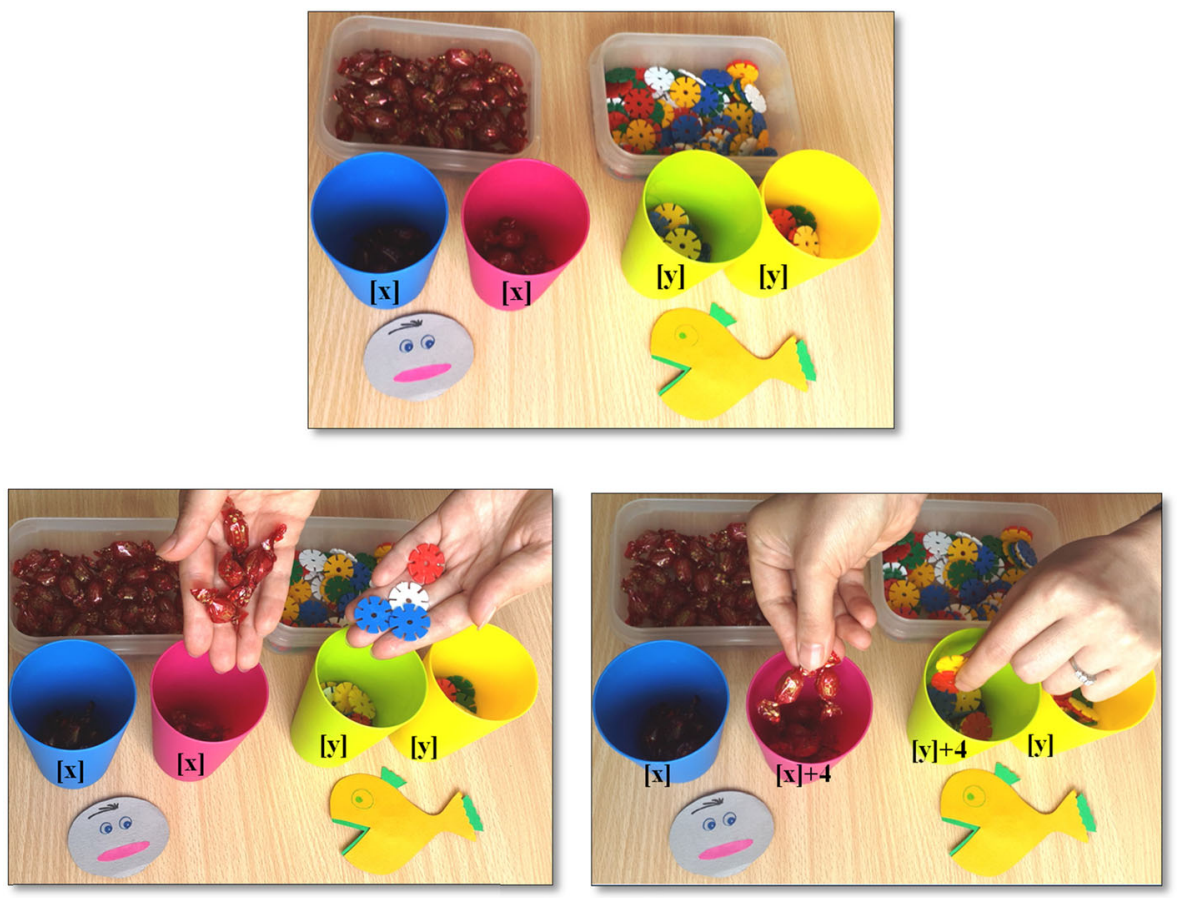

Fig. 4 Task 5 
Task 5 asks the child to compare $([x]+4)-2$ with $[x]$ and $([y]+4)-2$ with $[y]$. Both $[x]$ and $[y]$ are specific numbers (though kept unknown). Moreover, $[x]$ and $[y]$ are not necessarily equal to each other. In the transition of $([x]+4)-2$ to $[x]+2$ and $([y]+4)-$ 2 to $[y]+2$, there is no resort to specificity of $[x]$ and $[y]$, respectively. This is experiencing both transitions simultaneously as specific cases of the same structural change. It seems that there is a sense of generality involved in articulating, "Because you added four to them and removed two from them." This is to see that the total change would be valid for any starting number, though the range of "any" is determined by one's (here, Helen's) conception of the numbers involved.

\section{Concluding Remarks}

We presented and examined an approach to algebra that was based on arithmetic. In essence, like Carpenter et al. (2005) we advocated thinking relationally about equality. They have tried to foster it structurally. We attempted it operationally and extended previous research on early algebra to verbal mathematics. We interpreted the operational conception as the ability of transforming one structure into the other. Using a series of carefully designed tasks based on the idea of specularity (Asghari , 2012) we prompted 5-6-year-old children to think operationally in the absence of alphanumeric symbols. The focus was on the associative property.

We showed the possibility of developing an operational algebraic understanding of the associative property at the preschool level. In arithmetic, this appears as the ability of restructuring a given arithmetic expression to an equivalent one. In algebra, this is the ability of transforming an operation between an unknown and a known $([x]+4)-2$ into an operation between two knowns: $[x]+(4-2)$. Considering that these are what students need in mental and written methods of calculations (McCallum et al., 2011) and in a good part of algebra (Wasserman, 2014), our work highlights the surprisingly high level of what can be experienced by preschool children. More importantly, it is what the result of this study suggests for "the development of algebra":

In contrast to what Sfard and Linchevski (1994) and Caspi and Sfard (2012) say, our findings suggest that verbal and operational are not necessarily processual and bound to numeric computations. They have the potential to foster thinking relationally about equality with both known and unknown quantities. However, as always, there are some words of caution.

Due to the purely verbal nature of our study, we could not directly encourage "different ways of looking at the same number expression" that is necessary for the flexible use of the associative property in arithmetic. In a way, in our tasks, one structure collapses into the other without any trace. As soon as the numerals come into play, we can (must) encourage students to see operational transformations as different ways of structuring the same expression. Such awareness is the sine qua non of the structural conception of algebra and should be meaningfully linked to the operational conception via symbols (for some suggestions to link from the operational to the structural, see Khosroshahi \& Asghari, 2016; for the other way round, see Carpenter et al., 2003).

However, with great potential of symbolic representations comes great challenge. With the advent of written base-ten numerals comes the so-called left-to-right approach commonly used in early grades. With the equal sign comes its computational 
drive. With the letters for unknown quantities comes "the addictive power of algebraic manipulations" (Kieran \& Sfard, 1999, p. 15). Simply speaking, there is a long journey from operationally and verbally transforming $([x]+4)-2$ to $[x]+2$ (where $[x]$ is a specular number) to structurally and symbolically understanding the equality $x+4-2=x+2$ (where $x$ represents a variable). As one example among many, consider how in the verbal situation of our design, the operations were automatically attached to the operands (e.g. add four, remove two), all of which should be kept in memory (though not reported above, there were many times that we needed to remind children of the numbers used in the task at hand). In contrast, in a symbolic situation, the operations and operands are recorded, but children need to learn how to correctly interpret each and every one of them (that is not as easy as it might look; see Herscovics \& Linchevski, 1994).

What we did was as an exploration into where an algebraic journey of children might start. Surprisingly, this start can include an experience of working with unknown quantities through equality-preserving transformations. This also includes an affirmative answer to our research question that whether the idea of specularity can promote an operational algebraic understanding of associativity in the absence of alphanumeric symbols. However, we cannot claim that the participants of this study would be able to use what they experienced later on as primary school students. In fact, even in task 4 and in the middle of their exposure to many similar tasks, we could not unequivocally predict whether a child would restructure the given problem or not. Believing that providing an algebraic experience of arithmetic for students needs targeted tasks and, more importantly, an algebraic mentality, all we wish to claim is that we have offered both. There is still much to do before the tasks we used in a one-to-one situation can be adapted for the use in the classroom (for an attempt, see Khosroshahi \& Asghari, 2016), where an algebraic journey would not exist if an algebraic habit of mind is not infused into school curriculum (Blanton \& Kaput, 2003), whether we advocate an operational or structural approach.

Open Access This article is distributed under the terms of the Creative Commons Attribution 4.0 International License (http://creativecommons.org/licenses/by/4.0/), which permits unrestricted use, distribution, and reproduction in any medium, provided you give appropriate credit to the original author(s) and the source, provide a link to the Creative Commons license, and indicate if changes were made.

\section{References}

Asghari, A. H. (2012). Specularity in algebra. For the learning of mathematics, 32(3), 34-38.

Blanton, M. L. \& Kaput, J. J. (2003). Developing elementary teachers" "algebraic eyes and ears". Teaching Children Mathematics, 10(2), 70-77.

Booth, L. R. (1989). A question of structure. In S. Wagner \& C. Kieran (Eds.), Research issues in the learning and teaching of Algebra (pp. 57-59). Reston, VA: NCTM.

Carpenter, T. P., Franke, M. L. \& Levi, L. (2003). Thinking mathematically: Integrating arithmetic and algebra in elementary school. Portsmouth, NH: Heinemann.

Carpenter, T. P., Levi, L., Franke, M. L. \& Zeringue, J. K. (2005). Algebra in elementary school: Developing relational thinking. ZDM-The International Journal on Mathematics Education, 37(1), 53-59.

Carpenter, T. P., Levi, L. \& Farnsworth, V. (2000). Building a foundation for learning algebra in the elementary grades [In Brief]. National center for improving student learning and achievement in mathematics and science, 2(1), 1-4. Retreived from http://ncisla.wceruw.org 
Carraher, D., Brizuela, B. M. \& Schliemann, A. D. (2000). Bringing out the algebraic character of arithmetic: Instantiating variables in addition and subtraction. In T. Nakahara \& M. Koyama (Eds.), Proceedings of the 24th Conference of the International Group for the Psychology of Mathematics Education (Vol. 2, pp. 145-152). Hiroshima, Japan: PME.

Caspi, S. \& Sfard, A. (2012). Spontaneous meta-arithmetic as a first step toward school algebra. International Journal of Educational Research, 51, 45-65.

Cobb, P., Confrey, J., diSessa, A., Lehrer, R. \& Schauble, L. (2003). Design experiment in educational research. Educational Researcher, 32(1), 9-13.

Fujii, T. \& Stephens, M. (2001). Fostering an understanding of algebraic generalisation through numerical expressions. In H. Chick, K. Stacey, J. Vincent \& J. Vincent (Eds.), Proceedings of the 12th Conference of the International Commission on Mathematics Instruction.1 (pp. 258-264). Melbourne, Australia: ICMI.

Geary, D. C. (2006). Development of mathematical understanding. In W. Damon, R. M. Lerner, D. Kuhn \& R. S. Siegler (Eds.), Handbook of child psychology: Cognition, perception, and language (6th ed., Vol. 2, pp. 777-810). Hoboken, NJ: Wiley.

Gray, E. M. \& Tall, D. O. (1994). Duality, ambiguity and flexibility: a proceptual view of simple arithmetic. Journal for Research in Mathematics Education, 25(2), 115-141.

Herscovics, N. \& Linchevski, L. (1994). A cognitive gap between arithmetic and algebra. Educational Studies in Mathematics, 27(1), 59-78.

Hudson, T. (1983). Correspondences and numerical differences between disjoint sets. Child Development, 54(1), 84-90.

Khosroshahi, L. G. \& Asghari, A. H. (2016). Using untouchables to make arithmetic structures touchable: the case of associative rule. Australian primary mathematics classroom, 21(1), 8-11.

Kieran, C. (1981). Concepts associated with the equality symbol. Educational Studies in Mathematics, 12, 317-326.

Kieran, C. (1989). The early learning of algebra: a structural perspective. In S. Wagner \& C. Kieran (Eds.), Research issues in the learning and teaching of algebra (pp. 33-56). Reston, VA: NCTM.

Kieran, C. (2014). What does research tell us about fostering algebraic thinking in arithmetic?. Retrieved from http://old.nctm.org/uploadedFiles/Research_News_and_Advocacy/Research/Clips_and_Briefs/Algebraic

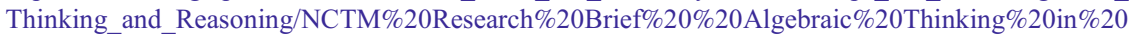
Arithmetic.pdf.

Kieran, C. \& Sfard, A. (1999). Seeing through symbols: the case of equivalent expressions. Focus on Learning Problems in Mathematics, 21(1), 1-17.

Larsen, S. (2010). Struggling to disentangle the associative and commutative properties. For the Learning of Mathematics, 3(1), 37-42.

Mason, J. \& Pimm, D. (1984). Generic examples: Seeing the general in the particular. Educational Studies in Mathematics, 15(3), 277-289.

Mason, J., Stephens, M. \& Watson, A. (2009). Appreciating mathematical structure for all. Mathematics Education Research Journal, 21(2), 10-32.

Mazur, B. (2008). When is one thing equal to another thing? In B. Gold \& R. Simons (Eds.), Proof and other dilemmas: mathematics and philosophy (pp. 221-242). Washington, DC: Mathematical Association of America.

McCallum, W., Zimba, J. \& Daro, P. (2011). Progressions for the common core state Standards in Mathematics. Retrieved from https://commoncoretools.files.wordpress.com/2012/02/ccss_progression nf_35_2011_08_12.pdf

Molina, M. \& Ambrose, R. (2008). From an operational to a relational conception of the equal sign. Thirds graders' developing algebraic thinking. Focus on Learning Problems in Mathematics, 30(1), 61-80.

Nunes, T. \& Bryant, P. (1996). Children doing mathematics. Oxford, United Kingdom: Blackwell.

Peled, I. \& Carraher, D. W. (2008). Signed numbers and algebraic thinking. In J. Kaput, D. W. Carraher \& M. Blanton (Eds.), Algebra in the early grades (pp. 303-328). Mahwah, NJ: Lawrence Erlbaum.

Radford, L. (2010). Elementary forms of algebraic thinking in young students. In M. M. F. Pinto \& T. F. Kawasaki (Eds.), Proceedings of the 34th Conference of the International Group for the Psychology of Mathematics Education (Vol. 4, pp. 73-80). Belo Horizonte, Brazil: PME.

Radford, L. (2014). The progressive development of early embodied algebraic thinking. Mathematics Education Research Journal, 26(2), 257-277.

Sfard, A. \& Linchevski, L. (1994). The gains and pitfalls of reification: the case of algebra. Educational Studies in Mathematics, 26, 191-228. 
Stephens, M. (2006). Describing and exploring the power of relational thinking. In P. Grootenboer, R. Zevenbergen \& M. Chinnappan (Eds.), Identities, Cultures and Learning Spaces: Proceedings of the 29th annual Conference of the Mathematics Education Research Group of Australasia (pp. 479-486). Canberra, Australia: MERGA.

Stephens, A. C., Knuth, E. J., Blanton, M. L., Isler, I., Gardiner, A. M. \& Marum, T. (2013). Equation structure and the meaning of the equal sign: The impact of task selection in eliciting elementary students' understandings. The Journal of Mathematical Behavior, 32, 173-182.

Wasserman, N. (2014). Introducing algebraic structures through solving equations: Vertical content knowledge for K-12 Mathematics teachers. PRIMUS, 24(3), 191-214. 